\title{
Dissociative mental states are canonically associated with decreased temporal theta activity on spectral analysis of the EEG
}

\author{
CHRISTA KRÜGER, MBBCh, MMed(Psych), MD, FCPsych(SA) \\ Department of Psychiatry, University of Pretoria; and Weskoppies Hospital, Pretoria, South Africa \\ PETER BARTEL, PhD \\ Neurophysiology Unit, Department of Neurology, University of Pretoria; and Steve Biko Academic Hospital, \\ Pretoria, South Africa \\ LIZELLE FLETCHER, BCom(Econometrics), Honours(Mathematical Statistics), MSc(Mathematical \\ Statistics), PhD(Statistics) \\ Department of Statistics, University of Pretoria, South Africa \\ Corresponding author: Prof Christa Krüger \\ Address: Department of Psychiatry, University of Pretoria, Weskoppies Hospital, Private Bag X113, Pretoria, \\ 0001, South Africa \\ Tel: +27 123199741 \\ Fax: +27 123199617 \\ E-mail: christa.kruger@up.ac.za \\ Word count: 4959
}

Grant support: Funding was received from the Research Committee of the Faculty of Health Sciences, University of Pretoria for the research assistant post, and from the Research Development Programme of the University of Pretoria. These sponsors had no role in the study design; in the collection, analysis and interpretation of data; in the writing of the report; or in the decision to submit the article for publication.

Acknowledgements: The authors acknowledge the assistance of Ms Lisinda Nel (research assistant) with the data collection; the clinical technologists of the Neurophysiology Laboratory, especially the late Ms Ria Heystek, for the EEG recordings; Dr Mike van der Linde for electronic data management; and Ms Barbara English for valuable editorial advice.

\section{Postal mailing addresses of authors:}

Prof Christa Krüger: Department of Psychiatry, University of Pretoria, Weskoppies Hospital, Private Bag X113, Pretoria, 0001, South Africa 
Prof Peter Bartel: Department of Neurology, University of Pretoria, Room 62289, Steve Biko Academic Hospital, Private Bag X169, Pretoria, 0001, South Africa

Dr Lizelle Fletcher: Department of Statistics, University of Pretoria, Private Bag X20, Hatfield, 0028, South Africa

\begin{abstract}
Quantitative electroencephalographic (QEEG) changes relating to dissociative experiences have only rarely been demonstrated and dissociative states were not quantified in those studies. The aim of this study was to explore concurrent associations between quantified dissociative states and QEEG spectral parameters, in particular theta activity, in psychiatric patients. Fifty psychiatric patients completed the State Scale of Dissociation (SSD) immediately after a 15-minute EEG recording. The EEG was assessed by conventional clinical visual analysis, as well as by quantitative (QEEG) spectral analysis. Canonical analysis was performed between the set of SSD subscale scores and these QEEG parameters: alpha-theta magnitude ratios, and relative as well as absolute theta magnitude obtained from right and left mid- to posterior-temporal and parieto-occipital derivations. The SSD transferred well to the present data in terms of reliability and internal-criterion-related validity. The SSD and DES correlated significantly $(r=0.73 ; p<0.001)$. Conventional EEG analysis identified 29 EEGs (58\%) as abnormal. The main abnormality in 23 EEGs was slowing, maximal temporally in half of these cases. Canonical analyses confirmed a statistically significant relationship between the dissociation variables (especially conversion and depersonalization symptoms) and the QEEG variables (especially relative theta magnitude in the temporal regions) ( $R=0.72 ; p=0.03$ for $S S D-Q E E G$ and $R=0.66 ; p=0.04$ for $D E S-Q E E G)$. Quantified dissociative mental states are positively canonically associated with decreased temporal theta activity and increased alpha-theta ratios on QEEG in psychiatric patients with a high tendency to dissociate. The potential implications of the dissociationtheta-alpha relationship for understanding normal attentional processes need to be studied further.
\end{abstract}

Keywords: Dissociation; dissociative mental states; quantitative electro-encephalography (QEEG); spectral analysis; theta amplitude; alpha-theta ratio; canonical correlation analysis; neurophysiology

In the context of a proliferation of research in the last few decades, it is slowly becoming clear how the psychological process of dissociation comes about and how it works at the neurophysiological level.

The concept 'dissociation' has been used in mainstream psychiatry/psychology for an involuntary disruption of the normal integration of conscious awareness and control over one's mental processes (Spiegel et al., 2011). Distinctions have been made between 
normal/pathological dissociation, and between dimensional/categorical concepts of dissociation (Spiegel et al., 2011), but an exploration of these distinctions falls outside the scope of this article. A more functional description that accounts for the neurobiological basis of dissociation is the one rendered by Felmingham et al. (2008) according to which dissociation is a regulatory strategy for coping with extreme arousal through hyperinhibition of limbic regions - a strategy most active during the conscious processing of threat.

Notwithstanding recent advances in the use of functional magnetic resonance imaging (fMRI), positron emission tomography (PET) and single-photon emission computerized tomography (SPECT) to study dissociation and related responses (Bremner et al., 1999a,b; Daniels et al., 2012; Felmingham et al., 2008; Lanius et al., 2002,2005; Mickleborough et al., 2011; Sar, Unal, \& Ozturk, 2007), another area in which the neurophysiology of dissociation has been studied, is electroencephalography (EEG).

Studies using conventional visual EEG analysis - not quantitative electroencephalography (QEEG) - have suggested an association between trait dissociation and temporal theta activity (Ray, Moraga, \& Faith, 1994; Spiegel \& Vermutten, 1994). Others have demonstrated an association between dissociation and epileptiform EEG activity (Coons, Bowman, \& Milstein, 1988; Schulz et al., 1995). However, these studies quantified neither dissociative states, nor the EEG.

QEEG changes relating to dissociative trait experiences have only rarely been demonstrated: an association between trait dissociation and theta power in undergraduate students (Giesbrecht, Jongen, Smulders, \& Merckelbach, 2006) and in patients with borderline personality disorder who felt no pain during a cold pressor test (Russ, Campbell, Kakuma, Harrison, \& Zanine, 1999); increased theta power in highly hypnotizable students, 
especially during hypnosis (Sabourin, Cutcomb, Crawford, \& Pribram, 1990); and increased beta, alpha or delta power in patients with multiple personality disorder during induction of alter personalities (Cocker, Edwards, Anderson, \& Meares, 1994; Lapointe, Crayton, DeVito, Fichtner, \& Konopka, 2006). Other studies have demonstrated a trend towards lefthemispheric lateralization in non-clinical individuals with inconsistent handedness and high trait dissociation (Ashworth, Ciorciari, \& Stough, 2008); a negative relationship between interregional EEG cross-correlation indices and trait dissociation in patients with paranoid schizophrenia (Bob, Susta, Glaslova, \& Boutros, 2010); and reduced complexity of the EEG associated with narrowing of attention during interview-assisted evocation of dissociative symptoms in a patient with an anxiety disorder and non-specific encephalopathy due to a motorcycle accident (Bob, Golla, Epstein, Konopka, 2011).

However, dissociative states were not quantified in any of the abovementioned studies.

The distinction between trait and state phenomena has long been regarded as potentially pivotal in the study of the etiology of dissociation, but has been neglected (Bedard-Gilligan \& Zoellner, 2012). Those authors recommend distinguishing between trait dissociation, state dissociation, peritraumatic dissociation and dissociation in response to traumatic reminders.

In this article, the focus is on acute dissociative states and the QEEG changes that accompany such dissociative states. Following on the previous findings of a link between trait dissociation and theta activity as mentioned above, the aim of this study was to explore concurrent associations between quantified dissociative states and QEEG parameters, in particular theta activity, in psychiatric patients. 


\section{METHODS}

\section{Design}

Fifty psychiatric patients completed a trait measure of dissociation - the Dissociative Experiences Scale (DES) (Bernstein \& Putnam, 1986). They also underwent a routine-type EEG recording and completed a state measure of dissociation - the State Scale of Dissociation (SSD) (Krüger \& Mace, 2002) immediately afterwards. The EEG was assessed by conventional clinical visual analysis and QEEG spectral analysis. The relationship between the dissociation variables and the EEG variables was analyzed statistically.

The objectives of the study were to:

- Determine if a statistically significant relationship exists between spontaneous dissociative mental states and certain QEEG variables, particularly theta activity; and

- Reconfirm the validity and reliability of the SSD as a measure of dissociative mental states.

\section{Setting and participants}

Convenience sampling was used at a specialist psychiatric hospital and a general academic hospital to recruit 50 adult psychiatric patients with a history of a high tendency to dissociate. Because of the frequent comorbidity between dissociative symptoms/disorders and depressive-, psychotic- and other psychiatric disorders, recruitment was done on the basis of

a history of clinically prominent dissociative symptoms rather than on diagnosis. The assumption was that high dissociators would be more likely to experience spontaneous dissociative states at the time of the study measurements than low dissociators. 


\section{Instruments and procedures}

Participants completed the 28-item self-report DES (Bernstein \& Putnam, 1986) at baseline to confirm their psychiatric history of a high tendency to dissociate. They also completed the psychometrically validated 56-item self-report SSD (Krüger \& Mace, 2002) immediately after an EEG recording to measure the intensity of these spontaneous dissociative experiences at the time of completing the scale: derealization, depersonalization, identity confusion, identity alteration, conversion, amnesia and hypermnesia.

A 15-minute EEG recording was done by trained neurophysiology staff for each participant sitting upright in a chair. The 10/20 system for electrode placement and a digital 32-channel Nihon-Kohden-Neurofax-EEG-2100 system were used. The montage used consisted of standard longitudinal anterior-posterior electrode chains constituting 18 channels, plus two dedicated channels to record eye movements - i.e. a 20-channel recording. The low cut-off filter was fixed at TC 0.1 second and the high cut-off filter at $35 \mathrm{~Hz}$.

Blinding was achieved in that the technical staff were unaware of the subjects' diagnoses or questionnaire scores. Muscle artifact was limited by recording the EEG with eyes closed in restful wakefulness. Epoch markers were manually set to exclude eye or other movements that could cause EEG artifacts. Vigilance was maintained by auditory stimulation when any of the following criteria for the onset of drowsiness was met: appearance of slow eye movements or theta activity, or attenuation of alpha rhythm.

The entire EEG recording was visually analyzed in the conventional clinical way. EEG deviations from normality were graded I(mild), II(moderate) or III(marked) according to an unpublished modification of the Mayo Clinic classification system (Department of Neurology, 1991). 
For the quantitative EEG analyses, editing was performed to exclude artifacts, although some muscle contamination of temporal derivations might have remained. The first 3.5-4 minutes of nearly-artifact-free EEG without signs of drowsiness were digitized at a sampling rate of 200/s. The Fast Fourier Transform was performed and the results summarized in four frequency bands: delta $(1.5-4 \mathrm{~Hz})$, theta $(4-8 \mathrm{~Hz})$, alpha $(8-13 \mathrm{~Hz})$, beta(13$30 \mathrm{~Hz}$ ). The magnitude of each waveband constituted the basic digital EEG data. Beta magnitudes were excluded from the calculation of the relative percentages of delta, theta and alpha activity to reduce the impact of possible medication effects. The software used was EEGFOCUS, version 2 (Megis Software GmbH, Munich, Germany).

The spectral analysis resulted in 18 EEG derivations/electrode pairs, e.g., P4-O2. Each derivation produced six absolute, six relative and two ratio variables. The total QEEG variables were 252 per subject. To reduce this large number we focused on theta activity on the grounds of previous studies (Introduction; Krüger, 1999). Relative theta magnitude and alpha-theta ratios $(\%)$ were calculated to reduce the high inter-individual variation in absolute EEG magnitude $(\mu \mathrm{V})$.

The EEG variable selection was further guided by the temporal involvement in dissociation on the conventional EEG analysis and the literature (Introduction). Hence, right and left mid- to posterior-temporal derivations (T4-T6; T3-T5) were chosen. Two parietooccipital derivations (P4-O2; P3-O1) were also included because these are least affected by muscle contamination. 


\section{Analyses}

A canonical analysis - a generalization of, or multivariate multiple regression (Sherry \& Henson, 2005) - was performed between the set of SSD-subscale scores and these QEEG spectral parameters: alpha-theta magnitude ratios and relative theta magnitude obtained from right and left mid- to posterior-temporal and parieto-occipital derivations. A similar analysis was performed for the set of DES-factor scores with these QEEG parameters. Canonical correlation analysis determines the primary independent latent dimensions that relate two sets of variables to one another. The nature of this relationship can be inferred by interpreting the relative contribution of each variable to the extracted canonical functions. Canonical analysis allows the extraction of more than one set of linear combinations of variables that are correlated, thereby providing the number of dimensions needed to account for the relationship (Sherry \& Henson, 2005).

Because of the limitation that relative theta magnitude cannot distinguish between an actual increase or decrease in theta activity as opposed to a decrease or increase in one or more of the other wavebands, the canonical analyses were subsequently repeated using the absolute theta values instead of the relative theta values, to determine if that would yield different results. This substitution of absolute values for relative values was done for both the SSD-QEEG- and the DES-QEEG canonical analyses.

To assess possible confounding variables, T-tests, ANOVAs, Pearson correlations and subgroup-labeled scatter plots were performed. For the confirmation of the reliability of the SSD, Cronbach's alpha was calculated for each subscale. Subscale-total Pearson correlations reflected internal-criterion-related validity of the 7-subscale structure of the SSD. The convergent validity of the SSD was examined through SSD-DES Pearson correlations. 


\section{Ethical considerations}

This study was approved by the Research Ethics Committee of the Faculty of Health Sciences, University of Pretoria. Written informed consent was obtained from participants after adequately explaining the study's procedures to them.

\section{RESULTS}

Table 1 summarizes clinical and demographic characteristics of the 50 study participants. Their psychiatric diagnoses included dissociative/conversion- $(\mathrm{n}=18 ; 36 \%)$, psychotic$(\mathrm{n}=12 ; 24 \%)$, and mood-/other disorders $(\mathrm{n}=20 ; 40 \%)$. Nobody suffered from epilepsy or psychogenic-non-epileptic seizures. Of the 50 participants, 41(82\%) used some medication; $38(76 \%)$ a combination of medications; $27(54 \%)$ an antipsychotic; $21(42 \%)$ an SSRI antidepressant; and 16(32\%) a benzodiazepine.

The descriptive statistics of the SSD and DES scores, absolute and relative theta magnitudes, alpha-theta ratios and visual EEG analysis are summarized in Table 2. The mean DES score of the participants was 33.84, confirming their high frequency of dissociative symptoms.

Conventional EEG analysis identified 29 EEGs(58\%) as abnormal (Table 2). The main abnormality in 23 of these EEGs was excessive slow wave activity, occurring maximally in the temporal areas in half of these cases. One of the patients with abnormal right-temporal slowing also had epileptiform activity there; another had right-posteriortemporal isolated sharp waves. None of them had previously been diagnosed with epilepsy.

Table 3 displays the results of the reliability and validity analyses. The internal consistency analyses yielded satisfactorily high Cronbach alpha values (all $>0.86$ ). With 
TABLE 1. Demographic and clinical characteristics of study population $(N=50)$

\begin{tabular}{|c|c|c|c|c|c|}
\hline & & $\mathrm{N}$ & $\%$ & Mean & Std dev \\
\hline \multirow[t]{2}{*}{ Gender } & Male & 14 & 28 & & \\
\hline & Female & 36 & 72 & & \\
\hline \multirow[t]{3}{*}{ Age (years) } & All & 50 & 100 & 37.80 & 11.13 \\
\hline & Male & 14 & 28 & 35.07 & 9.85 \\
\hline & Female & 36 & 72 & 38.86 & 11.54 \\
\hline \multirow{10}{*}{$\begin{array}{l}\text { Psychiatric } \\
\text { disorder }\end{array}$} & Conversion & 12 & 24 & & \\
\hline & Schizophrenia / schizo-affective & 12 & 24 & & \\
\hline & Major depressive & 8 & 16 & & \\
\hline & Dissociative & 6 & 12 & & \\
\hline & Bipolar & 5 & 10 & & \\
\hline & Personality & 3 & 6 & & \\
\hline & Somatoform & 1 & 2 & & \\
\hline & Post-traumatic stress & 1 & 2 & & \\
\hline & Obsessive-compulsive & 1 & 2 & & \\
\hline & Substance related & 1 & 2 & & \\
\hline \multirow{10}{*}{$\begin{array}{l}\text { Current } \\
\text { medication }\end{array}$} & None & 9 & 18 & & \\
\hline & Antipsychotic only & 1 & 2 & & \\
\hline & Antidepressant only & 2 & 4 & & \\
\hline & Combination of medications & 38 & 76 & & \\
\hline & Antipsychotic & 27 & 54 & & \\
\hline & Antidepressant & 28 & 56 & & \\
\hline & SSRI & 21 & 42 & & \\
\hline & Mood stabilizer & 16 & 32 & & \\
\hline & Benzodiazepine & 16 & 32 & & \\
\hline & Anticholinergic & 16 & 32 & & \\
\hline
\end{tabular}


TABLE 2. Dissociation and EEG descriptive statistics $(\mathrm{N}=50)$

\begin{tabular}{|c|c|c|c|}
\hline Measure & Subscale/sub-measure & $\begin{array}{r}\text { Mean } \\
\text { score/intensity }\end{array}$ & $\begin{array}{l}\text { Standard } \\
\text { deviation }\end{array}$ \\
\hline \multirow[t]{8}{*}{ SSD } & Derealization & 2.53 & 2.77 \\
\hline & Depersonalization & 2.63 & 2.75 \\
\hline & Identity confusion & 2.74 & 2.73 \\
\hline & Identity alteration & 1.90 & 2.16 \\
\hline & Conversion & 1.64 & 2.27 \\
\hline & Amnesia & 1.66 & 2.10 \\
\hline & Hypermnesia & 2.15 & 2.42 \\
\hline & Total SSD score $(\max =9)$ & 2.20 & 2.15 \\
\hline DES & Mean DES score $(\max =100)$ & 33.84 & 22.12 \\
\hline \multirow[t]{12}{*}{ QEEG } & Mean absolute right-temporal $\theta$ magnitude & $7.98 \mu \mathrm{V}$ & 5.16 \\
\hline & Mean absolute left-temporal $\theta$ magnitude & $7.54 \mu \mathrm{V}$ & 4.73 \\
\hline & Mean absolute right-posterior $\theta$ magnitude & $7.99 \mu \mathrm{V}$ & 5.11 \\
\hline & Mean absolute left-posterior $\theta$ magnitude & $7.88 \mu \mathrm{V}$ & 5.18 \\
\hline & Relative right-temporal $\theta$ magnitude & $17.51 \%$ & 6.80 \\
\hline & Relative left-temporal $\theta$ magnitude & $17.76 \%$ & 6.55 \\
\hline & Relative right-posterior $\theta$ magnitude & $19.32 \%$ & 6.45 \\
\hline & Relative left-posterior $\theta$ magnitude & $18.80 \%$ & 6.28 \\
\hline & Right-temporal $\alpha / \theta$ ratio & 1.61 & 0.54 \\
\hline & Left-temporal $\alpha / \theta$ ratio & 1.51 & 0.47 \\
\hline & Right-posterior $\alpha / \theta$ ratio & 1.74 & 0.63 \\
\hline & Left-posterior $\alpha / \theta$ ratio & 1.85 & 0.70 \\
\hline Visual & Normal EEG & $n=21(42 \%)$ & \\
\hline \multirow{4}{*}{$\begin{array}{l}\text { EEG } \\
\text { analysis }\end{array}$} & Abnormal EEG & $n=29(58 \%)$ & \\
\hline & Grade I & $n=8(16 \%)$ & \\
\hline & Grade II & $n=14(28 \%)$ & \\
\hline & Grade III & $\mathrm{n}=7(14 \%)$ & \\
\hline
\end{tabular}


TABLE 3. Reliability and validity of the State Scale of Dissociation (SSD) ( $N=50)$

Reliability / Internal consistency of the SSD:

\begin{tabular}{llll} 
& SSD items & Subscale title & Cronbach's Alpha \\
\hline 1 & Items 1-8 & Derealization & 0.934 \\
2 & Items 9-16 & Depersonalization & 0.929 \\
3 & Items 17-24 & Identity confusion & 0.915 \\
4 & Items 25-32 & Identity alteration & 0.883 \\
5 & Items 33-40 & Conversion & 0.913 \\
6 & Items 41-46 & Amnesia & 0.864 \\
7 & Items 47-56 & Hypermnesia & 0.922 \\
\hline
\end{tabular}

Internal-criterion-related validity (SSD subscale-total correlations):

\begin{tabular}{llll} 
& SSD items & Subscale title & $\begin{array}{l}\text { Pearson correlation } \\
\text { coefficient }\end{array}$ \\
\hline 1 & Items 1-8 & Derealization & $0.881^{*}$ \\
2 & Items 9-16 & Depersonalization & $0.950^{*}$ \\
3 & Items 17-24 & Identity confusion & $0.882^{*}$ \\
4 & Items 25-32 & Identity alteration & $0.841^{*}$ \\
5 & Items 33-40 & Conversion & $0.825^{*}$ \\
6 & Items 41-46 & Amnesia & $0.796^{*}$ \\
7 & Items 47-56 & Hypermnesia & $0.889^{*}$ \\
\hline
\end{tabular}

Convergent validity with the DES (SSD-DES correlations):

\begin{tabular}{llll} 
& SSD items & Subscale title & $\begin{array}{l}\text { Pearson correlation } \\
\text { coefficient }\end{array}$ \\
\hline 1 & Items 1-8 & Derealization & $0.638^{*}$ \\
2 & Items 9-16 & Depersonalization & $0.730^{*}$ \\
3 & Items 17-24 & Identity confusion & $0.601^{*}$ \\
4 & Items 25-32 & Identity alteration & $0.716^{*}$ \\
5 & Items 33-40 & Conversion & $0.494^{*}$ \\
6 & Items 41-46 & Amnesia & $0.655^{*}$ \\
7 & Items 47-56 & Hypermnesia & $0.651^{*}$ \\
& & & \\
& Items 1-56 & Total SSD & $0.733^{*}$ \\
\hline
\end{tabular}

${ }^{*} p$-value $<0.001$ 
regard to internal-criterion-related validity, the SSD subscale-total Pearson's correlation coefficients were all large $(0.79-0.95)$ and statistically highly significant $(p<0.001)$. Concerning convergent validity, the SSD and DES correlated significantly $(r=0.73 ; \mathrm{p}<0.001)$.

Table 4 summarizes the results of the canonical analyses. For the SSD-EEG canonical analysis, the individual canonical loadings of each SSD subscale with the SSD variate, as well as their cross-loadings with the QEEG variate, are ranked in decreasing order of magnitude, to indicate the relative importance of each variable in the SSD-QEEG relationship; and likewise for the QEEG variables. Similar rankings were done for the DESEEG canonical analysis.

FIGURE 1. Scatter plot of canonical SSD-QEEG relationship

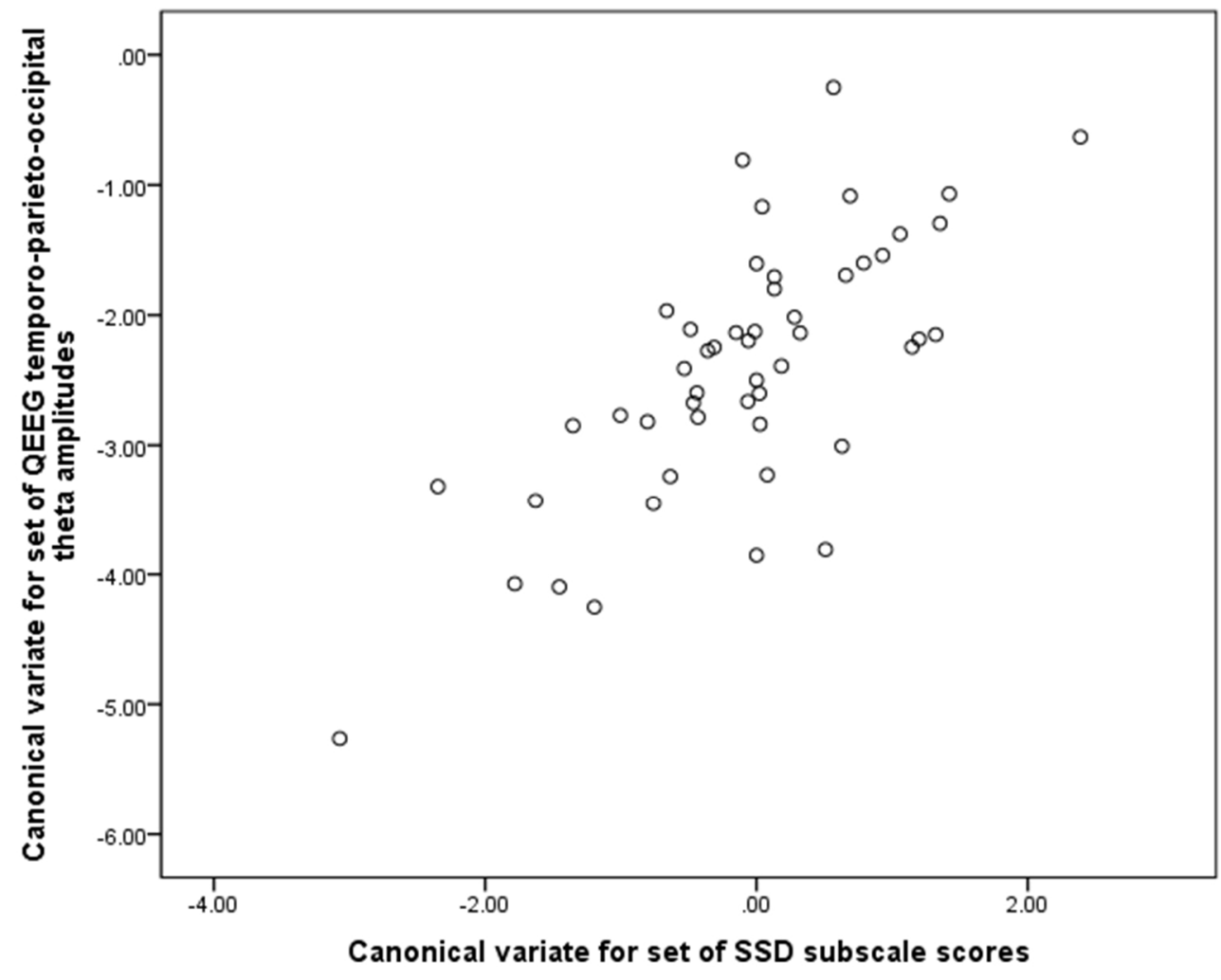


TABLE 4. Dissociation-EEG canonical analysis

SSD-EEG canonical correlation:

Root 1: $\mathrm{R}=0.72$; Chi-square $=77.62 ; p=0.03$; redundancy $=26.04 \%$

SSD variables: Canonical correlation with set of $Q E E G$ variables

\begin{tabular}{lrrr} 
SSD subscale & $\begin{array}{r}\text { Cross loading with } \\
\text { QEEG variate (rank) }\end{array}$ & $\begin{array}{r}\text { Canonical loading } \\
\text { with own variate } \\
\text { (rank) }\end{array}$ & $\begin{array}{r}\text { Canonical weight } \\
\text { (rank) }\end{array}$ \\
\hline Conversion & $0.337(1)$ & $0.469(1)$ & $0.976(3)$ \\
Depersonalization & $0.232(2)$ & $0.322(2)$ & $1.012(2)$ \\
Amnesia & $0.153(3)$ & $0.213(3)$ & $-0.412(6)$ \\
Identity alteration & $0.110(4)$ & $0.154(4)$ & $0.148(7)$ \\
Identity confusion & $-0.105(5)$ & $-0.145(5)$ & $-1.479(1)$ \\
Derealization & $0.086(6)$ & $0.120(6)$ & $0.510(5)$ \\
Hypermnesia & $-0.006(7)$ & $-0.009(7)$ & $-0.608(4)$ \\
\hline
\end{tabular}

QEEG variables: Canonical correlation with set of SSD variables

\begin{tabular}{lrrr} 
QEEG variable & $\begin{array}{c}\text { Cross loading with } \\
\text { SSD variate (rank) }\end{array}$ & $\begin{array}{r}\text { Canonical loading } \\
\text { with own variate } \\
\text { (rank) }\end{array}$ & $\begin{array}{r}\text { Canonical weight } \\
\text { (rank) }\end{array}$ \\
\hline Relative R-temp $\theta$ & $-0.240(1)$ & $-0.333(1)$ & $-1.926(2)$ \\
Relative L-temp $\theta$ & $-0.216(2)$ & $-0.301(2)$ & $0.291(6)$ \\
R-temp $\alpha / \theta$ ratio & $-0.140(3)$ & $-0.195(3)$ & $-2.212(1)$ \\
Relative L-post $\theta$ & $-0.131(4)$ & $-0.181(4)$ & $0.196(7)$ \\
Relative R-post $\theta$ & $-0.121(5)$ & $-0.168(5)$ & $0.814(5)$ \\
L-post $\alpha / \theta$ ratio & $0.114(6)$ & $0.158(6)$ & $0.961(3)$ \\
R-post $\alpha / \theta$ ratio & $0.040(7)$ & $0.056(7)$ & $0.062(8)$ \\
L-temp $\alpha / \theta$ ratio & $0.029(8)$ & $0.040(8)$ & $0.830(4)$ \\
\hline
\end{tabular}

DES-EEG canonical correlation:

Root 1: $\mathrm{R}=0.66$; Chi-square $=37.12 ; p=0.04$; redundancy $=36.5 \%$

DES variables: Canonical correlation with set of QEEG variables

$\begin{array}{crrr}\text { Cross loading with } & \text { Canonical loading } & \text { Canonical weight } \\ \text { QEEG variate (rank) } & \text { with own variate } & \text { (rank) }\end{array}$

\begin{tabular}{|c|c|c|c|}
\hline Dep-/derealization & $0.625(1)$ & $0.947(1)$ & $0.753(1)$ \\
\hline Amnestic dissociation & $0.591(2)$ & $0.896(2)$ & $0.528(2)$ \\
\hline Absorption/imaginative & $0.481(3)$ & $0.729(3)$ & $-0.255(3)$ \\
\hline \multicolumn{4}{|c|}{ QEEG variables: Canonical correlation with set of DES variables } \\
\hline QEEG variable & $\begin{array}{l}\text { Cross loading with } \\
\text { DES variate (rank) }\end{array}$ & $\begin{array}{r}\text { Canonical loading } \\
\text { with own variate } \\
\text { (rank) }\end{array}$ & $\begin{array}{r}\text { Canonical weight } \\
\text { (rank) }\end{array}$ \\
\hline L-temp $\alpha / \theta$ ratio & $0.484(1)$ & $0.734(1)$ & $0.472(7)$ \\
\hline Relative L-temp $\theta$ & $-0.407(2)$ & $-0.617(2)$ & $-0.786(5)$ \\
\hline L-post $\alpha / \theta$ ratio & $0.389(3)$ & $0.589(3)$ & $1.615(3)$ \\
\hline R-temp $\alpha / \theta$ ratio & $0.378(4)$ & $0.573(4)$ & $0.139(8)$ \\
\hline Relative R-post $\theta$ & $-0.366(5)$ & $-0.554(5)$ & $-1.920(1)$ \\
\hline Relative R-temp $\theta$ & $-0.345(6)$ & $-0.522(6)$ & $0.727(6)$ \\
\hline Relative L-post $\theta$ & $-0.340(7)$ & $-0.515(7)$ & $1.531(4)$ \\
\hline R-post $\alpha / \theta$ ratio & $0.269(8)$ & $0.408(8)$ & $-1.858(2)$ \\
\hline
\end{tabular}


The canonical analyses demonstrate a statistically significant direct relationship between the set of dissociative variables and the set of QEEG spectral parameters. The relationship is such that increased dissociation is positively correlated with decreased relative theta magnitude (and hence higher alpha-theta ratios) (Table 4). For the SSD-QEEG analysis, the canonical correlation coefficient is $\mathrm{R}=0.72(p=0.03)$ and the redundancy $26 \%$; i.e., about a quarter of the variance in the one set of variables can be accounted for by the variate of the other set (Figure 1). The canonical correlation in the SSD-QEEG analysis is predominantly associated with conversion, depersonalization and amnesia, on the one hand, and low relative temporal theta magnitude, on the other. The QEEG variate represents a contrast between low theta activity and corresponding high alpha-theta ratios. For the DES-QEEG analysis, $\mathrm{R}=0.66(p=0.04)$; redundancy $36.5 \%$ (Figure 2 ). For both analyses, only one canonical correlation was significant, hence no further dimensions were needed to explain the relationship between SSD/DES and QEEG. 
FIGURE 2. Scatter plot of canonical DES-QEEG relationship

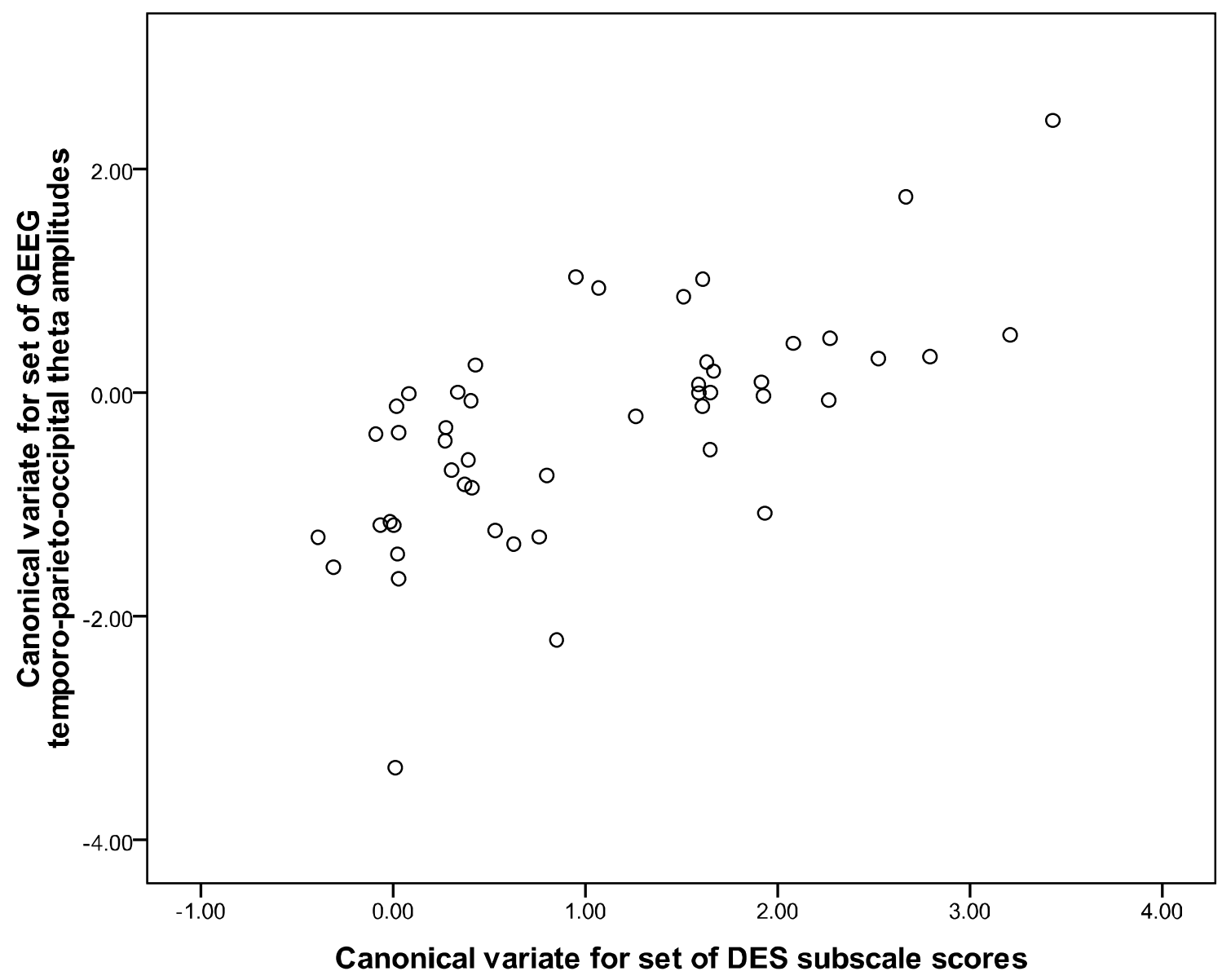

The canonical analyses that were subsequently repeated using absolute instead of relative theta magnitude yielded similar results. For the SSD-QEEG analysis, $\mathrm{R}=0.76(p=0.084)$; redundancy $16.2 \%$. For the DES-QEEG analysis, $\mathrm{R}=0.61(p=0.014)$; redundancy $32.2 \%$.

The analyses for possible confounding variables yielded significant results with regard to age, psychiatric diagnosis and abnormality of the EEG on visual analysis. With respect to age, younger patients dissociated more than older patients. Concerning psychiatric diagnosis, patients with dissociative-, conversion-, mood- and other disorders dissociated 
more than patients with psychotic disorders. With regard to abnormality of the EEG on visual analysis, patients with a normal EEG dissociated more than those with an abnormal EEG.

Medication use on the whole was not a confounding variable in the dissociationQEEG relationship on the basis of the statistical tests. Nevertheless, patients using an SSRI showed significantly higher dissociation scores than patients using other medications. There was no evidence that gender might be a confounding variable.

\section{DISCUSSION}

To our knowledge, this study is the first to demonstrate that quantified dissociative states are canonically and concurrently associated with temporal theta activity on spectral analysis of the EEG. The significant canonical correlation indicates that increased dissociation is positively correlated with decreased theta activity (and hence higher alpha-theta ratios). The canonical analysis accounts for the close relationships within the set of dissociative state variables and the close relationships within the set of QEEG spectral parameters. Moreover, the significant canonical correlation represents a robust statistical signal given the de facto diluted nature of the data, and illustrates the importance of applying appropriate statistical tests for multivariate data.

Interpretation of relationship between dissociation, theta activity and alpha-theta ratio

In order to interpret the decreased theta magnitude and increased alpha-theta ratio during dissociative states, we draw on previous studies on cognition in dissociation, particularly those on attention. 
The relationship between dissociation and attention has been studied by Freyd and coworkers using a series of Stroop tasks in undergraduate students scoring high or low on the DES (Freyd, Martorello, Alvarado, Hayes, \& Christman, 1998; DePrince \& Freyd, 1999, 2001). The first, standard Stroop task indicated disrupted attentional abilities in high dissociators (Freyd et al., 1998). In the second, extended Stroop design, under the divided attentional conditions the high trait dissociators performed better than the low dissociators, suggesting that multi-tasking facilitates the process of dissociating aversive stimuli (DePrince \& Freyd, 1999). Subsequent directed forgetting tasks demonstrated that during divided attention, the high trait dissociators forgot more of the trauma words in comparison with the low trait dissociators (DePrince \& Freyd, 2001; Giesbrecht, Lynn, Lilienfeld, \& Merckelbach, 2008). These findings of a link between dissociative tendencies and attention were supported by a recent study in 54 undergraduate female students, where Olsen \& Beck (2012) demonstrated that those with high DES scores showed less attention and poorer recall for the trauma stimuli than for the neutral stimuli, and similarly in comparison with the low DES group - under divided attention conditions.

Notwithstanding the developing picture that dissociation only happens effectively when attention is divided, it is a shortcoming that the abovementioned and numerous other previous studies of cognition in dissociation did not use a state measure of dissociation, nor were they supplemented by neurophysiological measurements such as EEG or QEEG (Giesbrecht et al., 2008). The extensive literature review by Giesbrecht et al. (2008) also demonstrated that previous studies on cognitive processes in dissociation mostly relied on either a diagnosis of a dissociative disorder or on self-report ratings of trait dissociation; and 
in the case of the latter, on extreme group designs that excluded the middle range of dissociation.

In contrast, the present study demonstrates the benefits of applying a state measure of dissociation, as well as a design that includes the whole range of intensity of dissociation. However, attention was not measured in the present study. It therefore remains difficult to interpret the relationship between dissociation, theta activity, alpha-theta ratio and attention in this study.

It might be helpful to refer to Cooper, Croft, Dominey, Burgess, \& Gruzelier (2003), who investigated the relationship between QEEG alpha activity and attention in normal subjects, comparing matched internally and externally directed tasks. Their study does not support the conventional idea of alpha activity reflecting cortical idling or inactivity. Instead, Cooper et al. (2003) demonstrated a clear relationship between alpha and internally directed attention, as well as between alpha and active inhibition of non-task relevant cortical areas.

Theta activity (particularly a reduction in theta-related intertrial phase locking around event-related potentials) has also been implicated in conditions of distracted attention (Ponjavic-Conte, Dowdall, Hambrook, Luczac, \& Tata, 2012). In another recent study, Nigbur, Ivanova, \& Stürmer (2011) demonstrate theta power enhancement related to the recruitment of cognitive control in situations of interference or conflicts in the stream of information processing. These studies seem to suggest that decreased theta activity might possibly be related in some way to divided attention. In addition, theta activity has been linked to working memory processes (Schmiedt, Brand, Hildebrandt, \& Basar-Eroglu, 2005; Klimesch, Schack, \& Sauseng, 2005). 
A full review of the literature on attention and working memory falls outside the scope of this article. However, a preliminary interpretation of our findings in the light of the above studies on attention might be that the decreased theta activity and increased alpha-theta ratio during dissociative states may reflect a state of increased attention, possibly divided attention, and an increased capacity for dual task performance.

\section{Confounding variables}

The identification in the present study of age, psychiatric diagnosis and abnormality of the EEG on visual analysis as potential confounding variables in the dissociation-QEEG relationship suggests that demographic and clinical variables may be important determinants of the degree of patients' dissociation. The finding that younger patients dissociated more than older patients is consistent with the literature (Giesbrecht et al., 2008). Although the patients with dissociative- or conversion disorders were not the only ones who dissociated in this study, psychiatric diagnosis played a role in how intensely and frequently patients dissociated. Furthermore, the finding that patients with a normal EEG dissociated more than those with an abnormal (slowed) EEG is in line with the main finding that increased dissociation is associated with decreased theta activity. The dissociation-theta canonical relationship was strong enough that, despite the slowing of the EEG on visual analysis in $58 \%$ of the patients, increased dissociation remained significantly associated with decreased theta activity.

Medication might also have played a confounding role, despite the non-significant statistical analysis for confounders. On the one hand, the inclusion of patients treated with medications that cause EEG slowing, may have resulted in a confounding effect. If so, 
however, the slowing effect of the medication was not strong enough to obscure the significant canonical dissociation-theta-alpha relationship. On the other hand, the methodological elimination of possible medication-induced beta activity might have limited the confounding effect of medication. Furthermore, the higher dissociation scores of patients using SSRIs are not surprising, as emotional detachment, derealization and depersonalization are common side effects of SSRIs (Price, Cole, \& Goodwin, 2009).

The issue of the nature of the relationship between dissociation and epileptiform EEG activity is also highlighted again because of the localization in this study of the EEG theta activity in the temporal lobes, and because two of the patients showed temporal epileptiform activity and temporal isolated sharp waves respectively on visual EEG analysis (albeit without clinical signs of a seizure). These findings are in line with previous research demonstrating that temporal lobe seizure activity can produce dissociative symptoms possibly via common pathophysiological processes that may include a kindling effect and chaos in brain neural networks (Bob, 2007).

\section{Validity and reliability of the SSD}

This study provides additional evidence for the validity and reliability of the SSD as a measure of state dissociation. The SSD transferred well to the present data in terms of reliability, internal-criterion-related validity, and convergent validity with the DES. Furthermore, the significant canonical correlations of the QEEG with both the SSD and DES support their convergent validity and suggest that the two scales tap into the same construct of dissociation. 


\section{Limitations}

Methodological limitations of this study include that it was not possible to exclude all temporal muscle artifact. Some residual artifact might have resulted in increased fast wave activity in the EEG, which might have affected all percentages of EEG waveband activity. There does not seem to be a ready solution to this problem in awake patients.

Another possible limitation relates to the choice of bipolar derivations to display the QEEG data in this study. This choice sought to reduce the possible impact of particularly muscle artifact, since bipolar derivations may be less contaminated than, especially, ear reference derivations. Moreover, the use of bipolar derivations is likely to be advantageous for comparing visual and spectral EEG findings, as done here. On the down side, the choice in this study of bipolar derivations means that neighbouring electrodes are similarly influenced by slow activities; however, extra-cephalic reference electrodes, e.g., earlobe references, could have caused other problems.

Moreover, in this study theta activity was targeted on the basis of previous studies. However, the dissociation-QEEG canonical relationship might also be studied with respect to the other wavebands, particularly delta activity, to enrich the interpretation of this study's findings.

Furthermore, the small sample size and subgroups made it impossible to divide the data into subsets for separate analyses in order to ascertain, e.g., whether the patients with dissociative disorders had a stronger dissociation-QEEG canonical relationship than patients with other diagnoses. Similarly, it was not possible to analyze separately the data of the younger vs older patients, unmedicated vs medicated patients, or abnormal EEGs vs normal EEGs. 
In addition, the nature of this study does not allow for an evaluation of the corticolimbic inhibition model of dissociation (Lanius et al., 2002; Lanius, Bluhm, Lanius, \& Pain, 2006). Even though the SSD measures symptoms of primary, secondary and tertiary dissociation, the scalp EEG might not record with sufficient accuracy electrical potentials in deep structures such as the hippocampus and amygdala. The challenge remains to integrate the unique (temporally localizing) findings from QEEG studies with those from fMRI, SPECT and PET studies.

\section{Future research directions}

Further studies of the QEEG correlates of dissociative states appear necessary, if the abovementioned methodological limitations could be addressed and if further crossfertilization could take place between the fields of dissociation and cognitive-and-affective neuroscience.

Whereas spontaneous dissociative states were studied here, future QEEG studies might investigate induced dissociative states (Simone-Reinders et al., 2006) or dissociative states during trauma-related tasks (Bedard-Gilligan \& Zoellner, 2012). Moreover, future studies might investigate additional QEEG derivations, especially prefrontal/frontal derivations (Forrest, 2001) and occipital derivations (Daniels et al., 2012), as well as delta activity. The roles of the abovementioned potential confounding variables should also be investigated further.

Furthermore, psychological testing of attention after traumatic and neutral films as performed by Olsen \& Beck (2012), or the Stroop methodology used by Freyd and DePrince 
and coworkers (1998, 1999, 2001), or other working memory paradigms (Schmiedt et al., 2005; Klimesch et al., 2005) might be supplemented with QEEG and the SSD.

Alternatively, the relationship between dissociative states and attention might be studied further by analysing the complexity of the EEG (Bob, Golla, Epstein, \& Konopka, 2011). Or the QEEG methodology might be extended to estimate functional connectivity of theta activity according to the methods used by Koenig, Lehmann, Saito, Kuginuki, Kinoshita, and Koukkou (2001).

In addition, the mixed-methods methodology used by Meltzer, Fonzo, \& Constable (2009) to study the relationship between EEG theta power, hippocampal fMRI activation and memory, might hold promise for future studies of cortico-limbic interaction involved in the link between dissociation, theta activity, attention and working memory.

\section{Conclusions}

Quantified dissociative mental states are positively canonically associated with decreased temporal theta activity and increased alpha-theta ratio on QEEG in psychiatric patients with a high tendency to dissociate. This study provides additional evidence for the validity and reliability of the SSD as a measure of state dissociation. Further studies are needed to confirm the generalizability of the dissociation-theta canonical association, and to elucidate the link/s between the psychological process of dissociation and other cognitive processes. For example, the potential implications of the dissociation-theta-alpha relationship for understanding normal attentional processes need to be studied further. 


\section{REFERENCES}

Ashworth, J., Ciorciari, J., Stough, C., (2008). Psychophysiological correlates of dissociation, handedness, and hemispheric lateralization. The Journal of Nervous and Mental Disease, 196(5), 411-6.

Bedard-Gilligan, M., Zoellner, L.A., (2012). Dissociation and memory fragmentation in post-traumatic stress disorder: An evaluation of the dissociative encoding hypothesis. Memory, 20(3), 277-99.

Bernstein, E.M., Putnam, F.W., (1986). Development, reliability, and validity of a dissociation scale. The Journal of Nervous and Mental Disease, 174(12), 727-35.

Bob, P., (2007). Dissociation, forced normalization and dynamic multi-stability of the brain. Neuroendocrinology Letters, 28(3), 231-46.

Bob, P., Susta, M., Glaslova, K., Boutros, N.N., (2010). Dissociative symptoms and interregional EEG crosscorrelations in paranoid schizophrenia. Psychiatry Research, 177, 37-40.

Bob, P., Golla, M., Epstein, P., Konopka, L., (2011). EEG complexity and attentional processes related to dissociative states. Clinical EEG \& Neuroscience, 42(3), 175-9.

Bremner, J.D., Staib, L.H., Kaloupek, D., Southwick, S.M., Soufer, R., Charney, D.S., (1999a). Neural correlates of exposure to traumatic pictures and sound in Vietnam combat veterans with and without posttraumatic stress disorder: A positron emission tomography study. Biological Psychiatry, 45, 806816.

Bremner, J.D., Narayan, M., Staib, L.H., Southwick, S.M., McGlashan, T., Charney, D.S., (1999b). Neural correlates of memories of childhood sexual abuse in women with and without posttraumatic stress disorder. Am J Psychiatry, 156(11), 1787-1795.

Cocker, K.I., Edwards, G.A., Anderson, J.W., Meares, R.A., (1994). Electrophysiological changes under hypnosis in multiple personality disorder: A two-case exploratory study. Australian Journal of Clinical and Experimental Hypnosis, 22(2), 165-76.

Coons, P.M., Bowman, E.S., Milstein, V., (1988). Multiple personality disorder: A clinical investigation of 50 cases. The Journal of Nervous and Mental Disease, 176(9), 519-27.

Cooper, N.R., Croft, R.J., Dominey, S.J.J., Burgess, A.P., Gruzelier, J.H., (2003). Paradox lost? Exploring the role of alpha oscillations during externally vs. internally directed attention and the implications for idling and inhibition hypotheses. International Journal of Psychophysiology, 47, 65-74.

Daniels, J.K., Coupland, N.J., Hegadoren, K.M., Rowe, B.H., Densmore, M., Neufeld, R.W., Lanius, R.A., (2012). Neural and behavioral correlates of peritraumatic dissociation in an acutely traumatized sample. Journal of Clinical Psychiatry, 73(4), 420-6.

Department of Neurology, Mayo Clinic and Mayo Foundation, (1991). Clinical examinations in Neurology, 6th edn., St Louis, Mosby: Year Book Inc., pp. 354-390.

DePrince, A.P., Freyd, J.J., (1999). Dissociative tendencies, attention, and memory. Psychological Science, $10(5), 449-452$. 
DePrince, A.P., Freyd, J.J., (2001). Memory and dissociative tendencies: The roles of attentional context and word meaning in a directed forgetting task. Journal of Trauma and Dissociation, 2(2), 67-82.

Felmingham, K., Kemp, A.H., Williams, L., Falconer, E., Olivieri, G., Peduto, A., \& Bryant, R., (2008). Dissociative responses to conscious and non-conscious fear impact underlying brain function in posttraumatic stress disorder. Psychological Medicine, 38, 1771-80.

Forrest, K.A., (2001). Toward an etiology of dissociative identity disorder: A neurodevelopmental approach. Consciousness and Cognition, 10, 259-293.

Freyd, J.J., Martorello, S.R., Alvarado, J.S., Hayes, A.E., Christman, J.C., (1998). Cognitive environments and dissociative tendencies: Performance on the Standard Stroop Task for high versus low dissociators. Applied Cognitive Psychology, 12, S91-S103.

Giesbrecht, T., Jongen, E.M.M., Smulders, F.T.Y., Merckelbach, H., (2006). Dissociation, resting EEG, and subjective sleep experiences in undergraduates. The Journal of Nervous and Mental Disease, 194(5), $362-8$.

Giesbrecht, T., Lynn, S.J., Lilienfeld, S.O., Merckelbach, H., (2008). Cognitive processes in dissociation: An analysis of core theoretical assumptions. Psychological Bulletin, 134(5), 617-647.

Klimesch, W., Schack, B., Sauseng, P. (2005). The functional significance of theta and upper alpha oscillations. Experimental Psychology, 52(2), 99-108.

Koenig, T., Lehmann, D., Saito, N., Kuginuki, T., Kinoshita, T., Koukkou, M. (2001). Decreased functional connectivity of EEG theta-frequency activity in first-episode, neuroleptic-naïve patients with schizophrenia: Preliminary results. Schizophrenia Research, 50(1-2), 55-60.

Krüger, C., (1999). The State Scale of Dissociation: Development, psychometric validation, and application in a study of concurrent electro-encephalographic correlates. MD Thesis, University of Warwick, United Kingdom. http://wrap.warwick.ac.uk/38291/

Krüger, C., Mace, C.J., (2002). Psychometric validation of the State Scale of Dissociation (SSD). Psychology and Psychotherapy: Theory, Research and Practice (formerly the British Journal of Medical Psychology), 75(1), 33-51.

Lanius, R.A., Williamson, P.C., Boksman, K., Densmore, M., Gupta, M., Neufeld, R.W.J., Gati, J.S., Menon, R.S., (2002). Brain activation during script-driven imagery induced dissociative responses in PTSD: A functional magnetic resonance imaging investigation. Biological Psychiatry, 52, 305-311.

Lanius, R.A., Williamson, P.C., Bluhm, R.L., Densmore, M., Boksman, K., Neufeld, R.W.J., Gati, J.S., Menon, R.S., (2005). Functional connectivity of dissociative responses in posttraumatic stress disorder: A functional magnetic resonance imaging investigation. Biological Psychiatry, 57, 873-884.

Lanius, R.A., Bluhm, R., Lanius, U., \& Pain, C., (2006). A review of neuroimaging studies in PTSD: Heterogeneity of response to symptom provocation. Journal of Psychiatric Research, 40, 709-729. 
Lapointe, A.R., Crayton, J.W., DeVito, R., Fichtner, C.G., Konopka, L.M., (2006). Similar or disparate brain patterns? The intra-personal EEG variability of three women with multiple personality disorder. Clinical EEG and Neuroscience, 37(3), 235-42.

Meltzer, J.A., Fonzo, G.A., Constable, R.T. (2009). Transverse patterning dissociates human EEG theta power and hippocampal BOLD activation. Psychophysiology, 46(1), 153-62.

Mickleborough, M.J.S., Daniels, J.K., Coupland, N.J., Kao, R., Williamson, P.C., Lanius, U.F., Hegadoren, K., Schore, A., Densmore, M., Stevens, T., Lanius, R.A., (2011). Effects of trauma-related cues on pain processing in posttraumatic stress disorder: An fMRI investigation. $J$ Psychiatry Neurosci, 36(1), 6-14.

Nigbur, R., Ivanova, G., Stürmer B. (2011). Theta power as a marker for cognitive interference. Clinical Neurophysiology, 122(11), 2185-94.

Olsen, S.A., Beck, J.G., (2012). The effects of dissociation on information processing for analogue trauma and neutral stimuli: A laboratory study. Journal of Anxiety Disorders, 26, 225- 232.

Ponjavic-Conte, K.D., Dowdall, J.R., Hambrook, D.A., Luczak, A., Tata, M.S. (2012). Neural correlates of auditory distraction revealed in theta-band EEG. NeuroReport, 23(4), 240-5.

Price, J., Cole, V., Goodwin, G.M., (2009). Emotional side-effects of selective serotonin reuptake inhibitors: Qualitative study. The British Journal of Psychiatry, 195, 211-7.

Ray, W., Moraga, R., Faith, M., (1994). Psychometric and psychophysiological studies of hypnotisability and dissociation. International Journal of Clinical and Experimental Hypnosis, XLII(4), 481.

Russ, M.J., Campbell, S.S., Kakuma, T., Harrison, K., Zanine, E., (1999). EEG theta activity and pain insensitivity inself-injurious borderline patients. Psychiatry Research, 89, 201-14.

Sabourin, M.E., Cutcomb, S.D., Crawford, H.J., Pribram, K., (1990). EEG correlates of hypnotic susceptibility and hypnotic trance: Spectral analysis and coherence. International Journal of Psychophysiology, 10, $125-42$.

Sar, V., Unal, S.N., Ozturk, E., (2007). Frontal and occipital perfusion changes in dissociative identity disorder. Psychiatry Research: Neuroimaging, 156, 217-223.

Schmiedt, C., Brand, A., Hildebrandt, H., Basar-Eroglu, C. (2005). Event-related theta oscillations during working memory tasks in patients with schizophrenia and healthy controls. Cognitive Brain Research, 25(3), 936-47.

Schulz, R., Lüders, H.O., Noachtar, S., et al., (1995). Amnesia of the epileptic aura. Neurology, 45, 231-35.

Sherry, A., Henson, R.K. (2005). Conducting and interpreting canonical correlation analysis in personality research: A user-friendly primer. Journal of Personality Assessment, 84(1), 37-48.

Simone-Reinders, A.A.T., Nijenhuis, E.R.S., Quak, J., Korf, J., Haaksma, J., Paans, A.M.J., Willemsen, A.T.M., Den Boer, J.A., (2006). Psychobiological characteristics of dissociative identity disorder: A symptom provocation study. Biological Psychiatry, 60, 730-40.

Spiegel, D., Vermutten, E., (1994). Physiological correlates of hypnosis and dissociation. In D. Spiegel, (Ed.), Dissociation: culture, mind, and body. Washington: American Psychiatric Press. 
Spiegel, D., Loewenstein, R.J., Lewis-Fernández, R., Sar, V., Simeon, D., Vermetten, E., Cardeña, E., Dell, P.F., (2011). Dissociative disorders in DSM-5. Depression and Anxiety, 28, 824-52. 